\title{
Circularly Polarized Laser Fields, with Different $Z$, Including Non-Zero Initial Momentum
}

\author{
V.M. RistiĆ* ${ }^{*}$ T.B. MiladinoviĆ And J.M. StevanoviĆ \\ Faculty of Science, Kragujevac University, Radoja Domanovića 12, 34000 Kragujevac, Serbia \\ (Received July 27, 2010; revised version December 24, 2010; in final form January 18, 2011)
}

\begin{abstract}
In this paper, by estimating the influence of different atom charge $Z$ to the transition rate in tunnel ionization of atoms in strong laser fields we are devoloping further the observations from our earlier work. That is in the process of tunnel ionization including non-zero momentum into calculation of the transition rate gives result in lower transition rates for ejecting electrons from atoms by low-frequency laser fields, indicating that much of the photons are engaged in transferring energy to the free electron and thus unable to contribute to the effect of ionization. This is a conclusion that needs further experimental testing, which would clarify the mechanisms of tunnel ionization.
\end{abstract}

PACS: 32.80.-t, 32.80.Fb, 32.80.Rm

\section{Introduction}

It is definitely shown [1-10] that for low frequency laser fields one of the mostly used models is based on the tunneling regime: external field, due to its long duration, leads to the formation of the barrier through which electron can tunnel out. Among theories using this model the ADK-theory [1] is widely recognized. This theory was developed for linearly polarized laser fields, but it can be extended to the circularly polarized lasers, also $[2,3,9]$.

As we have recently shown that the momentum of the ejected electron influences the transition rate in the case of ADK-theory [2, 7-9], we are now examining how the change of atom charge $Z$ [8], when including non-zero momentum, contributes to transition rates for circularly polarized laser fields. Here, we are devoloping further the observations from our earlier work which included the ADK-theory. Namely, the process of tunnel ionization including non-zero momentum into calculation of the transition rate gives result in lower transition rates for ejecting electrons from atoms by low-frequency laser fields. All our observations indicate that much of the photons are engaged in transferring energy to the free electron and thus are unable to contribute to the effect of ionization.

Transition rate formula for circularly polarized field (in cases of zero momentum) was obtained earlier [3, 9] (atomic unit (a.u.) system $\hbar=m_{\mathrm{e}}=e=1$, is used here and throughout the paper).

\footnotetext{
* corresponding author; e-mail: ristic@kg.ac.rs
}

$$
\begin{aligned}
& W_{\mathrm{ADK}}^{\mathrm{cir}}=\left(\frac{3 F Z^{3}}{\pi n^{* 3}}\right)^{1 / 2}\left(\frac{4 Z^{3} \mathrm{e}}{F n^{* 4}}\right)^{2 n^{*}-1} \\
& \quad \times \exp \left(-\frac{2 Z^{3}}{3 F n^{* 3}}\right)
\end{aligned}
$$

where $Z$ is charge state, $F$ is field intensity, e is the logarithmic base not to be confused with elementary electric charge (which is 1 in the atomic system of units), and $n^{*}=Z / \sqrt{2 E}_{\mathrm{i}}$ is effective principal quantum number, $E_{\mathrm{i}}$ being the ionization potential.

In order to include the non-zero initial momentum into the expression (1), we used method of complex turning point, obtained from relation $E_{\mathrm{i}}(\tau)=E_{\mathrm{f}}(\tau)$, where $E_{\mathrm{i}}$ and $E_{\mathrm{f}}$ are energies of initial and final states of ejected electron, respectively $[2,6,11]$. After expanding trigonometry functions into power series, expression for non-zero initial momentum is calculated and included into exponential part of Eq. (1):

$$
\begin{gathered}
W_{\mathrm{pADK}}^{\mathrm{cir}}=\left(\frac{3 F Z^{3}}{\pi n^{* 3}}\right)\left(\frac{4 Z^{3} \mathrm{e}}{F n^{* 4}}\right)^{2 n^{*}-1} \\
\times \exp \left(-\frac{2 Z^{3}}{3 F n^{* 3}}-\frac{p^{2} \gamma^{3}}{3 \omega}\right) .
\end{gathered}
$$

Expression for momentum, which we obtained in paper $[2,7]$ is calculated introducing parabolic coordinates, by solving the Schrödinger equation for a charged particle in the Coulomb field $[12,13]$. It is found that expression for momentum is 


$$
p(\eta)=\sqrt{-\frac{1}{4}+\frac{1}{2 \eta}+\frac{1}{4 \eta^{2}}+\frac{F}{4} \eta},
$$

where $\eta$ is parabolic coordinate depending on the field intensity obtained from classical turning point $(\eta \sim 1 / F)$. $\eta$ could, in our case (3), be considered equivalent in its behavior to $p$. For example, $F \approx 10^{12} ; \eta=268.421$ (a.u. system). For greater fields, $\eta$ is decreasing, obviously.

Further, developing Eq. (3) into power series gives

$$
p(\eta)=\frac{1}{2}\left(\sqrt{F \eta-1}+\frac{1}{\eta \sqrt{F \eta-1}}+\ldots\right)
$$

Outside barrier $\eta>1 / F$.

Now we have introduced all necessary expressions for our further calculations. Next we shall present the new results that are obtained.

\section{Calculating the transition rates for circularly polarized laser fields including non-zero momentum, for different charge $Z$}

After that preparation we calculated transition rates for ejected electrons for various atomic charge $Z$, which is the new result first to be published here. The procedure is experimentally founded in [10]. Our results are shown on 3D and 2D graphs in Figs. 1-4. As parameters $\gamma$ and $n^{*}$ include in themselves ionization potential $E_{\mathrm{i}}$ : $\gamma=\omega \sqrt{2 E_{\mathrm{i}}} / F, n^{*}=Z / \sqrt{2 E_{\mathrm{i}}}$, we, as is done in experiments [10] and also analyzed in [9], define and calculate the change of ionization energy for each ejected electron. So graphs shown are depending on various $\eta[1,300]$, and on field intensities $I=10^{12}-10^{17} \mathrm{~W} / \mathrm{cm}^{2}$, which are fixed for each separate graph.

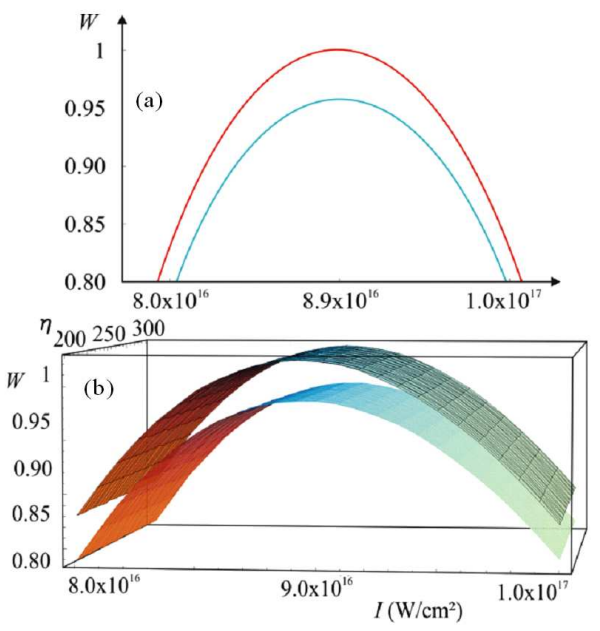

Fig. 1. Transition rates $W_{\mathrm{ADK}}^{\mathrm{cir}}$ and $W_{\mathrm{pADK}}^{\mathrm{cir}}$ dependence on field intensity, for zero initial momentum and non-zero initial momentum, respectively (enlarged parts), $Z=2, E_{2}=1.16$; (a) $\eta$ fixed at 190 (2D graph); (b) $\eta$ varying (3D graph).
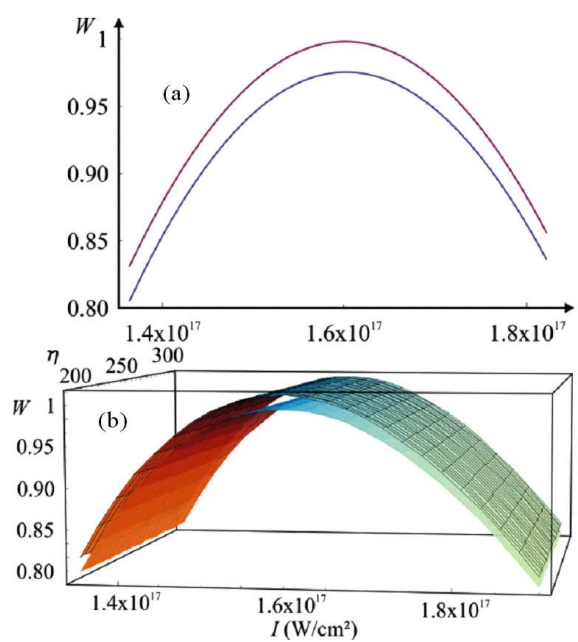

Fig. 2. Transition rates $W_{\mathrm{ADK}}^{\mathrm{cir}}$ and $W_{\mathrm{pADK}}^{\mathrm{cir}}$ dependence on field intensity, for zero initial momentum and non-zero initial momentum, respectively, $Z=3$, $E_{3}=1.68$; (a) $\eta$ fixed at 190 (2D graph); (b) $\eta$ varying (3D graph).

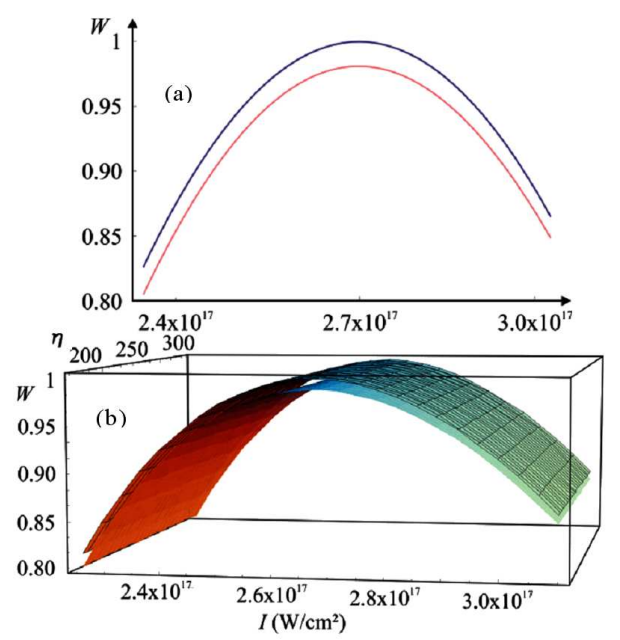

Fig. 3. Transition rates $W_{\mathrm{ADK}}^{\mathrm{cir}}$ and $W_{\mathrm{pADK}}^{\mathrm{cir}}$ dependence on field intensity, for zero initial momentum and non-zero initial momentum, respectively, $Z=4$, $E_{4}=2.24$; (a) $\eta$ fixed at 190 (2D graph); (b) $\eta$ varying (3D graph).

In order to mark the difference between the case of zero momentum of the ejected electron and non-zero momentum we have shown in each figure the transition rates maxima for both $W_{\mathrm{ADK}}^{\text {cir }}$ and $W_{\mathrm{pADK}}^{\text {cir }}$, depending on intensity of lasers and parabolic coordinate $\eta$, which is approximately proportional to $p(3)$, notifying the value of ion charge number $Z$, and the corresponding ionization energy (atomic unit system).

One should notice considerably large gap between the curves, for $Z=2$, indicating that the transition rate for ejected electrons with zero initial momentum and the 

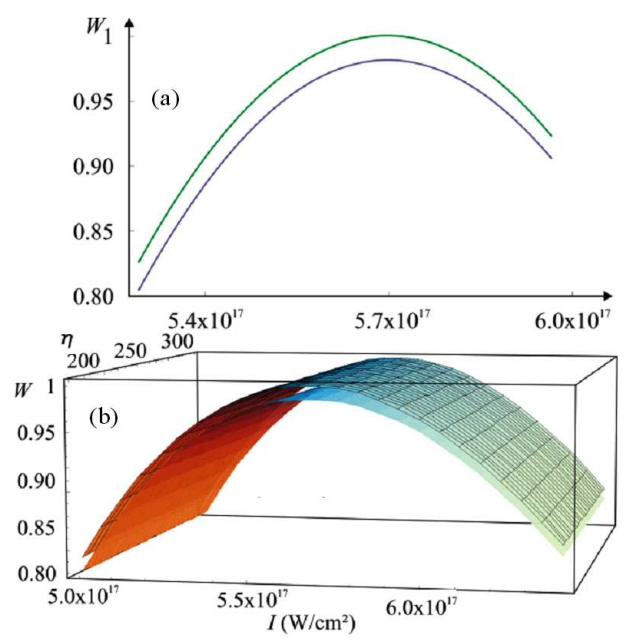

Fig. 4. Transition rates $W_{\mathrm{ADK}}^{\mathrm{cir}}$ and $W_{\mathrm{pADK}}^{\mathrm{cir}}$ dependence on field intensity, for zero initial momentum and non-zero initial momentum, respectively, $Z=5$, $E_{5}=3.04$; (a) $\eta$ fixed at 190 (2D graph); (b) $\eta$ varying (3D graph).

transition rate for electrons with non-zero momentum are different. The transition rate for electrons with non-zero momentum are much lesser as lots of photons are spent for increasing momentum.

For $Z=3$, the gap between curves is reduced, showing that the difference between the transition rate for ejected electrons with zero initial momentum and the transition rate for electrons with non-zero momentum are getting lesser, as $Z$ increases.

Here, for $Z=4$, the gap-effect is continuing to show itself, being smaller and smaller. See also the forthcoming figures.

Comparing Figs. 1-4 one can notice the greater $Z$, the lesser difference between two curves. It could be explained by the greater ionization energy needed for multiple ionized atoms.

Namely, as $Z$ is increasing, the influence of ejected electrons momentum on transition rates is decreasing, because much more photons from laser beam are used for overcoming the binding energy in the ion for greater $Z$. Therefore there is much less photons which could increase the momentum of ejected electrons, which can be seen from the lesser difference between corresponding curves. This is a prediction that should be tested experimentally, of course (for the linear polarization see [10]).

\section{Final remarks}

We have noticed that as $Z$ increases, the influence of non-zero momentum of ejected electrons decreases. It is due to the fact that more and more photons from the laser beam are used to overcome the binding energy in the multiply ionized atom, and there are lesser photons for increasing the initial momentum of ejected electrons.

Examining carefully Figs. 1-4 one can conclude that the greater $Z$, the lesser difference between two curves. It could be explained by the greater ionization energy needed for multiple ionized atoms, so there are less photons left to be transferred to the ejected electron, and the effect of non-zero initial momentum is damped.

Also, though maxima are at the same laser intensities, in the case of zero momentum the transition rate is greater (which can be seen especially in 2D graphs).

As mentioned earlier in the text these are predictions of the theory which should be compared with experimental results, if any (in [10] the similar procedure for linearly polarized field is strongly confirmed by the experiment). That could clarify the mechanisms of the tunnel ionization of atoms in low frequency laser fields.

\section{Acknowledgments}

This work was supported in part by the Ministry of Science, Republic of Serbia (project 171021).

\section{References}

[1] V.M. Ammosov, N.B. Delone, V.P. Krainov, Sov. Phys.-JETP 64, 1191 (1986).

[2] V.M. Ristić, T.B. Miladinović, M.M. Radulović, Laser Phys. 18, 1183 (2008).

[3] N.B. Delone, V.P. Krainov, Phys. Usp. 41, 469 (1998).

[4] M. Brewczyk, M. Gajda, J. Phys. B, At. Mol. Opt. Phys. 21, L383 (1988).

[5] S. August, D. Strickland, D.D. Meyerhofer, S.L. Chin, J.H. Eberly, Phys. Rev. Lett. 63, 2212 (1989).

[6] V.P. Krainov, V.M. Ristić, Sov. Phys.-JETP 101, 1479 (1992).

[7] V.M. Ristić, T.B. Miladinović, M.M. Radulović, Acta Phys. Pol. A 112, 909 (2007).

[8] V.M. Ristić, J.M. Stevanović, Laser Phys. Lett. 4, 354 (2007).

[9] V.M. Ristić, T.B. Miladinović, M.M. Radulović, Acta Phys. Pol. A 116, 504 (2009).

[10] K. Yamakawa, Y. Akahane, Y. Ukuda, M. Aoyama, N. Inoue, H. Ueda, T. Utsumi, Phys. Rev. Lett. 92, 123001 (2004).

[11] V.M. Ristić, T.S. Premović, Kragujevac J. Sci. 25, 41 (2003).

[12] D. Bauer, Phys. Rev. A 55, 2180 (1997).

[13] D. Bauer, Theory of Laser-Matter Interaction, Max-Planck Institute, Heidelberg 2002. 